\title{
Hazarders of Smoking and Helicobacter pylori Infection on Gastric Mucosa among Egyptian Patients with Dyspepsia
}

\author{
Rasha I. Salama1, Mahmoud W. Emara², Shimaa M. El Sharawy ${ }^{3}$ \\ ${ }^{1}$ Department of Tropical Medicine, Faculty of Medicine, Zagazig University, Zagazig, Egypt \\ ${ }^{2}$ Department of Pathology, Faculty of Medicine, Zagazig University, Zagazig, Egypt \\ ${ }^{3}$ Department of Tropical Medicine and Infectious Diseases, Faculty of Medicine, Tanta University, Tanta, Egypt \\ Email: rashasalama1010@gmail.com,nahla.Elgamal@yahoo.com, shaimaa.elsharawy@med.tanta.edu.eg
}

How to cite this paper: Salama, R.I., Emara, M.W. and El Sharawy, S.M. (2021) Hazarders of Smoking and Helicobacter pylori Infection on Gastric Mucosa among Egyptian Patients with Dyspepsia. Open Journal of Gastroenterology, 11, 1-15. https://doi.org/10.4236/ojgas.2021.111001

Received: December 11, 2020

Accepted: January 23, 2021

Published: January 26, 2021

Copyright $\odot 2021$ by author(s) and Scientific Research Publishing Inc. This work is licensed under the Creative Commons Attribution International License (CC BY 4.0).

http://creativecommons.org/licenses/by/4.0/

\begin{abstract}
Background: Smoking is hazardous to almost any organ in the body and has a harmful effect on the gastric mucosa. Objective: The main goal of this study was to evaluate the synergistic effect of smoking and $H$. pylori infection on gastric mucosal among dyspeptic Egyptian patients. Patients and Methods: A cross-sectional study was conducted among 240 consecutive patients with dyspepsia who underwent upper endoscopy and histopathological examination of gastric biopsies at Zagazig and Tanta University Hospital with $H$. $p y$ lori-positivity assessment by stool antigen and rapid urease test. Results: There were 60 smoker patients and 180 non-smoker patients. Erosive gastritis was more prevalent in smoker patients than non-smoker patients $(46.7 \%$ versus $15.6 \%, \mathrm{p}=0.00)$. In $H$. pylori-positive patients ( $36.7 \%$ of all patients), smokers were more prevalent than non-smokers in the development of intestinal metaplasia $(20 \%$ versus $0 \%, \mathrm{p}=0.003)$, erosive gastritis $(80 \%$ versus $30 \%, \mathrm{p}=0.00)$, glandular atrophy $(20 \%$ versus $0 \%, \mathrm{p}=0.003)$ and reactive gastropathy ( $20 \%$ versus $0 \%, p=0.003)$. Conclusions: This study revealed that smoking may increase the prevalence of having gastric intestinal metaplasia, erosive gastritis, glandular atrophy and reactive gastropathy in $H$. pylori-positive Egyptian patients.
\end{abstract}

\section{Keywords}

Dyspepsia, Helicobacter pylori, Smoking, Egypt

\section{Introduction}

Dyspepsia is a medical disorder that is characterized by persistent or recurring 
upper abdominal pain [1], and typically is classified into organic part and functional part [2], which may be associated by other symptoms of the gastrointestinal tract, such as belching, vomiting, nausea, fullness and early satiety postprandial [3]. It is one of the most common issues for patients referring to the endoscopists [4]. In addition, for conditions such as peptic ulcers and gastric cancers, dyspepsia is frequently the first warning sign [5]. Previously, multiple variables have been seemed to be closely linked to symptoms of dyspepsia which involving age, gender, non-steroidal anti-inflammatory drugs and antacids usage, H. pylori Infection and smoking [6] [7] [8].

Helicobacter pylori is a gram-negative microaerophilic bacillus likely to be placed in the deep sections of the mucosal gel over the gastric mucosa or even between the mucous layer and the epithelium of the stomach [9]. In developing nations, H. Pylori is generally acquired in early childhood and can continue to exist if left untreated for a lifetime [10] [11]. Whereas the bacterium is assessed to be embedded in the gastric mucosa of half the global population, only about $15-20$ percent of colonised persons contributes to the development of such diseases [12]. Chronic $H$. infection Pylori activates the immune response of the host, induces active chronic inflammation and damage to the mucosa, giving rise to multifocal atrophic gastritis, glandular dysplasia, intestinal metaplasia and adenocarcinoma [13].

There are between $10^{14}$ and $10^{16}$ free radicals in cigarette smoke [14], which can induce the formation of reactive oxygen species which really cause extensive damage to tissue. Cigarette smoking has also been shown to increase the frequency and recurrence of peptic ulcer disease [15] and several studies have also shown a significant link between smoking and the threat of $H$. pylori infection, as well as the development of the disease [16]. Furthermore, prolonged cigarette smoking really seemed to alter mucus production by the gastric mucosa [17]. Although the association between smoking and atrophic gastritis (AG) and intestinal metaplasia (IM) remains controversial [18] [19], numerous researchers have associated cigarette consumption with an increased risk of AG, IM and dysplasia largely dependent on histopathologic diagnosis [20] [21]. This work aimed to determine the different endoscopic and histological changes in gastric mucosa promoted by smoking among $H$. pylori-positive and $H$. pylori-negative Egyptian patients with dyspepsia.

\section{Patients and Methods}

\subsection{Study Design and Setting}

A cross-sectional study was conducted among two hundred forty consecutive patients who presented with dyspepsia symptoms and had been attending endoscopic units of the Tropical Medicine Department at Zagazig University Hospital, Egypt and Tanta University Hospital, Egypt between August 2019 and March 2020. The patients who agreed to participate were enrolled in this study after taking informed written consent. Studied patients with dyspepsia were di- 
vided according to their current smoking status into two groups (smoker and non-smoker) and based on $H$. pylori status; patients were divided into $H$. pylori positive and $H$. pylori negative.

Inclusion criteria: Patients who presented with dyspepsia, were advised for upper GI endoscopic (UGIE) evaluation with gastric biopsies evaluation and agreed to participate included in the study. Smoker patients, in this study, must smoke more than 5 cigarettes in the day for at least 3 years before.

Exclusion criteria: Patients refused to participate in the study or to undergo UGIE; patients were previously diagnosed to have $H$. pylori infection; patients had history the use of certain medications (NSAIDs, H2-receptor antagonists, proton pump inhibitors, alcohol and antibiotics) one month ago prior the endoscope; $\mathrm{x}$ smoker not current were excluded from the study.

\subsection{Method}

All patients were subjected to history taking, clinical examination, laboratory investigation, abdominal ultrasonography, H. pylori stool antigen (Epitope Diagnostics, Inc. (EDI) Fecal Helicobacter pylori Antigen ELISA kit) at the time of endoscopy.

UGIE (Olympus CV-150 or Pentax EPM3500) was performed for all the patients with dyspepsia as an objective assessment for the diagnosis of causes was also performed.

Four gastric biopsies were performed for all the included patients. After the endoscopy the biopsy samples were transferred to the lab under an appropriate condition for histological examination. Microscopic slices of the gastric mucosa were stained with hematoxylineosin and Giemsa. All the samples were additionally evaluated by the rapid urease test (CLO test; Kimberly-Clark Ltd., Draper, Utah, USA) at least in one of two gastric locations (antrum or corpus) and the results were read up to 24 hours at room temperature as a part of histological diagnosis to confirm $H$. pylori-positive status when the test showed red-violet color.

\subsection{Ethical Considerations}

The research steps were explained and the permission for conducting the research was obtained by the ethics committee of the Faculty of Medicine, Zagazig University, Egypt. This study was performed in accordance with the Declaration of Helsinki, Good Clinical Practice and applicable regulatory requirements.

\subsection{Statistical Analysis}

Analysis of data was done using SPSS version 20 (Armonk, NY: IBM Corp). Data were presented as mean \pm SD if they were quantitative or percentage if they were qualitative. Comparisons between qualitative variables were done using the Chi-square test while quantitative variables were assessed using the Unpaired Student $\mathrm{t}$-test. Results were considered significant when $\mathrm{P}$-value $\leq 0.05$. 


\section{Results}

Overall, 240 patients with dyspepsia were enrolled during the study period. The mean age \pm SD was $44.18 \pm 6.9$ and the majority were male (91.7\%). H. pylori infection was detected in 88 patients. As regard smoking, only $25.0 \%$ were smoker (60 cases). (Table 1 )

Gastritis and reflux were the most common endoscopic findings in this study 208 (86.7\%) and 104 (43.3\%) cases respectively. (Table 2)

As regard histological examination, $51.7 \%$ of the patient had chronic inactive gastritis. $45 \%$ had chronic active gastritis, $23.3 \%$ had erosive gastritis, and no biopsies revealed dysplasia. (Table 3)

As Table 4 shows, there is a significant statistical relationship between smoking and histopathological findings including erosive gastritis. Also, smokers more significantly showed reflux, erosion, peptic ulcer and mucosal nodularity in gastric endoscopic examination ( $\mathrm{P}$-value $<0.01$ ).

In this study, patients with $H$. pylori infection were significantly higher regard age. There was a statistically significant relationship between $H$. pylori infection; detection of erosion in the gastric endoscopic examination and erosive gastritis in histological examination (P-value $<0.01$ ). (Table 5)

The additive effects of smoking on endoscopic and histo-pathological findings in $H$. pylori-positive patients is shown in Table 6, this patient group was significantly showed a picture of intestinal metaplasia, erosive gastritis, glandular atrophy and reactive gastropathy in their histological examination. Also, these patients significantly had more erosion and peptic ulcer during endoscopic examination $(\mathrm{P}$-value $<0.01)$.

Table 1. Distribution of dyspeptic patient according to demographic data, current H. pylori infection and smoking status.

\begin{tabular}{|c|c|c|c|}
\hline & & \multicolumn{2}{|c|}{ Age } \\
\hline \multicolumn{2}{|c|}{ Mean \pm SD } & \multicolumn{2}{|c|}{$44.18 \pm 6.9$} \\
\hline \multicolumn{2}{|c|}{ Median (Range) } & \multicolumn{2}{|c|}{$44.0(30-58)$} \\
\hline & & $\mathrm{N}$ & $\%$ \\
\hline \multirow{2}{*}{ Sex } & Male & 220 & 91.7 \\
\hline & Female & 20 & 8.3 \\
\hline \multirow{3}{*}{ Smoking } & Non smoker & 180 & 75.0 \\
\hline & Smoker & 60 & 25.0 \\
\hline & Total & 240 & 100.0 \\
\hline \multirow{3}{*}{ H. pylori } & $-\mathrm{VE}$ & 152 & 63.3 \\
\hline & $+\mathrm{VE}$ & 88 & 36.7 \\
\hline & Total & 240 & 100.0 \\
\hline
\end{tabular}


Table 2. Endoscopic findings among the studied dyspeptic patients.

\begin{tabular}{cccc}
\hline & & $\mathrm{N}$ & $\%$ \\
\hline Reflux & $-\mathrm{VE}$ & 136 & 56.7 \\
& $+\mathrm{VE}$ & 104 & 43.3 \\
\hline Hiatus hernia & $-\mathrm{VE}$ & 208 & 86.7 \\
& $+\mathrm{VE}$ & 32 & 13.3 \\
\hline Gastritis & $-\mathrm{VE}$ & 32 & 13.3 \\
& $+\mathrm{VE}$ & 208 & 86.7 \\
\hline Erosion & $-\mathrm{VE}$ & 184 & 76.7 \\
& $+\mathrm{VE}$ & 56 & 23.3 \\
\hline Peptic ulcer & $-\mathrm{VE}$ & 216 & 90.0 \\
& $+\mathrm{VE}$ & 24 & 10.0 \\
\hline Mucosal nodularity & $-\mathrm{VE}$ & 224 & 93.3 \\
& $+\mathrm{VE}$ & 16 & 6.7 \\
\hline & Total & 240 & 100.0 \\
\hline
\end{tabular}

Table 3. Histological findings among the studied dyspeptic patients.

\begin{tabular}{|c|c|c|c|}
\hline & & $\mathrm{N}$ & $\%$ \\
\hline & $-\mathrm{VE}$ & 132 & 55.0 \\
\hline \multicolumn{4}{|l|}{ Chronic active gastritis } \\
\hline & $+\mathrm{VE}$ & 108 & 45.0 \\
\hline \multirow{2}{*}{\multicolumn{4}{|c|}{ Chronic inactive gastritis }} \\
\hline & & & \\
\hline \multirow{2}{*}{\multicolumn{4}{|c|}{ Dysplasia }} \\
\hline & & & \\
\hline & $+\mathrm{VE}$ & 0 & 0.0 \\
\hline \multirow{2}{*}{ Intestinal metaplasia } & $-\mathrm{VE}$ & 232 & 96.7 \\
\hline & $+\mathrm{VE}$ & 8 & 3.3 \\
\hline \multirow{3}{*}{ Erosive gastritis } & $-\mathrm{VE}$ & 184 & 76.7 \\
\hline & & & \\
\hline & $+\mathrm{VE}$ & 56 & 23.3 \\
\hline \multirow{3}{*}{ Glandular atrophy } & $-\mathrm{VE}$ & 224 & 93.3 \\
\hline & & & \\
\hline & $+\mathrm{VE}$ & 16 & 6.7 \\
\hline \multirow{3}{*}{ Reactive gastropathy } & $-\mathrm{VE}$ & 232 & 96.7 \\
\hline & $+\mathrm{VE}$ & 8 & 3.3 \\
\hline & Total & 240 & 100.0 \\
\hline
\end{tabular}


Table 4. Comparison of endoscopic and histological finding between the smoker and non-smokers dyspeptic patients.

\begin{tabular}{|c|c|c|c|c|c|c|}
\hline & & & Non & Smoker & $t / X^{2}$ & $\mathrm{P}$ \\
\hline & Age & & $44.23 \pm 9.97$ & $42.93 \pm 7.47$ & 1.821 & 0.068 \\
\hline \multirow{4}{*}{ Sex } & \multirow{2}{*}{ Male } & $\mathrm{N}$ & 160 & 60 & & \\
\hline & & $\%$ & $88.9 \%$ & $100.0 \%$ & & \\
\hline & \multirow{2}{*}{ Female } & $\mathrm{N}$ & 20 & 0 & 5.87 & $0.009^{\star}$ \\
\hline & & $\%$ & $11.1 \%$ & $0.0 \%$ & & \\
\hline \multirow{4}{*}{ H. pylori } & \multirow{2}{*}{$-\mathrm{VE}$} & $\mathrm{N}$ & 112 & 40 & & \\
\hline & & $\%$ & $62.2 \%$ & $66.7 \%$ & & \\
\hline & \multirow{2}{*}{$+\mathrm{VE}$} & $\mathrm{N}$ & 68 & 20 & 0.383 & 0.536 \\
\hline & & $\%$ & $37.8 \%$ & $33.3 \%$ & & \\
\hline \multirow{4}{*}{ Reflux } & \multirow{2}{*}{$-\mathrm{VE}$} & $\mathrm{N}$ & 116 & 20 & & \\
\hline & & $\%$ & $64.4 \%$ & $33.3 \%$ & & \\
\hline & \multirow{2}{*}{$+\mathrm{VE}$} & $\mathrm{N}$ & 64 & 40 & 17.73 & $0.00^{* *}$ \\
\hline & & $\%$ & $35.6 \%$ & $66.7 \%$ & & \\
\hline \multirow{4}{*}{$\begin{array}{l}\text { Hiatus } \\
\text { hernia }\end{array}$} & \multirow{2}{*}{$-\mathrm{VE}$} & $\mathrm{N}$ & 156 & 52 & & \\
\hline & & $\%$ & $86.7 \%$ & $86.7 \%$ & & \\
\hline & \multirow{2}{*}{$+\mathrm{VE}$} & $\mathrm{N}$ & 24 & 8 & 0.00 & 1.00 \\
\hline & & $\%$ & $13.3 \%$ & $13.3 \%$ & & \\
\hline \multirow{4}{*}{ Gastritis } & \multirow{2}{*}{$-\mathrm{VE}$} & $\mathrm{N}$ & 16 & 16 & & \\
\hline & & $\%$ & $8.9 \%$ & $26.7 \%$ & & \\
\hline & \multirow{2}{*}{$+\mathrm{VE}$} & $\mathrm{N}$ & 164 & 44 & 12.31 & $0.00^{* *}$ \\
\hline & & $\%$ & $91.1 \%$ & $73.3 \%$ & & \\
\hline \multirow{4}{*}{ Erosion } & \multirow{2}{*}{$-\mathrm{VE}$} & $\mathrm{N}$ & 148 & 36 & & \\
\hline & & $\%$ & $82.2 \%$ & $60.0 \%$ & & \\
\hline & \multirow{2}{*}{$+\mathrm{VE}$} & $\mathrm{N}$ & 32 & 24 & 12.42 & $0.00^{* *}$ \\
\hline & & $\%$ & $17.8 \%$ & $40.0 \%$ & & \\
\hline \multirow{4}{*}{ Peptic ulcer } & \multirow{2}{*}{$-\mathrm{VE}$} & $\mathrm{N}$ & 168 & 48 & & \\
\hline & & $\%$ & $93.3 \%$ & $80.0 \%$ & & \\
\hline & \multirow{2}{*}{$+\mathrm{VE}$} & $\mathrm{N}$ & 12 & 12 & 8.88 & $0.003^{x}$ \\
\hline & & $\%$ & $6.7 \%$ & $20.0 \%$ & & \\
\hline \multirow{4}{*}{$\begin{array}{c}\text { Mucosal } \\
\text { nodularity }\end{array}$} & \multirow{2}{*}{$-\mathrm{VE}$} & $\mathrm{N}$ & 176 & 48 & & \\
\hline & & $\%$ & $97.8 \%$ & $80.0 \%$ & & \\
\hline & \multirow{2}{*}{$+\mathrm{VE}$} & $\mathrm{N}$ & 4 & 12 & 22.85 & $0.00^{* *}$ \\
\hline & & $\%$ & $2.2 \%$ & $20.0 \%$ & & \\
\hline \multirow{4}{*}{$\begin{array}{c}\text { Chronic active } \\
\text { gastritis }\end{array}$} & \multirow{2}{*}{$-\mathrm{VE}$} & $\mathrm{N}$ & 104 & 28 & & \\
\hline & & $\%$ & $57.8 \%$ & $46.7 \%$ & & \\
\hline & \multirow{2}{*}{$+\mathrm{VE}$} & $\mathrm{N}$ & 76 & 32 & 2.24 & 0.134 \\
\hline & & $\%$ & $42.2 \%$ & $53.3 \%$ & & \\
\hline
\end{tabular}




\section{Continued}

\begin{tabular}{|c|c|c|c|c|c|c|}
\hline \multirow{4}{*}{$\begin{array}{l}\text { Chronic } \\
\text { inactive } \\
\text { gastritis }\end{array}$} & \multirow{2}{*}{$-\mathrm{VE}$} & $\mathrm{N}$ & 76 & 40 & \multirow{4}{*}{10.76} & \multirow{4}{*}{$0.001^{\star *}$} \\
\hline & & $\%$ & $42.2 \%$ & $66.7 \%$ & & \\
\hline & \multirow{2}{*}{$+\mathrm{VE}$} & $\mathrm{N}$ & 104 & 20 & & \\
\hline & & $\%$ & $57.8 \%$ & $33.3 \%$ & & \\
\hline \multirow{4}{*}{$\begin{array}{l}\text { Intestinal } \\
\text { metaplasia }\end{array}$} & \multirow{2}{*}{$-V E$} & $\mathrm{~N}$ & 176 & 56 & & \\
\hline & & $\%$ & $97.8 \%$ & $93.3 \%$ & & \\
\hline & \multirow{2}{*}{$+\mathrm{VE}$} & $\mathrm{N}$ & 4 & 4 & 2.75 & 0.097 \\
\hline & & $\%$ & $2.2 \%$ & $6.7 \%$ & & \\
\hline \multirow{4}{*}{$\begin{array}{l}\text { Erosive } \\
\text { gastritis }\end{array}$} & \multirow{2}{*}{$-V E$} & $\mathrm{~N}$ & 152 & 32 & & \\
\hline & & $\%$ & $84.4 \%$ & $53.3 \%$ & & \\
\hline & \multirow{2}{*}{$+\mathrm{VE}$} & $\mathrm{N}$ & 28 & 28 & 24.34 & $0.00^{\star *}$ \\
\hline & & $\%$ & $15.6 \%$ & $46.7 \%$ & & \\
\hline \multirow{4}{*}{$\begin{array}{c}\text { Glandular } \\
\text { atrophy }\end{array}$} & \multirow{2}{*}{$-V E$} & $\mathrm{~N}$ & 168 & 56 & & \\
\hline & & $\%$ & $93.3 \%$ & $93.3 \%$ & & \\
\hline & \multirow{2}{*}{$+\mathrm{VE}$} & $\mathrm{N}$ & 12 & 4 & 0.00 & 1.00 \\
\hline & & $\%$ & $6.7 \%$ & $6.7 \%$ & & \\
\hline \multirow{4}{*}{$\begin{array}{c}\text { Reactive } \\
\text { gastropathy }\end{array}$} & \multirow{2}{*}{$-\mathrm{VE}$} & $\mathrm{N}$ & 176 & 56 & & \\
\hline & & $\%$ & $97.8 \%$ & $93.3 \%$ & & \\
\hline & \multirow{2}{*}{$+\mathrm{VE}$} & $\mathrm{N}$ & 4 & 4 & 2.75 & 0.097 \\
\hline & & $\%$ & $2.2 \%$ & $6.7 \%$ & & \\
\hline \multirow{2}{*}{\multicolumn{2}{|c|}{ Total }} & $\mathrm{N}$ & 180 & 60 & & \\
\hline & & $\%$ & $100.0 \%$ & $100.0 \%$ & & \\
\hline
\end{tabular}

Table 5. Comparison of endoscopic and histological finding between the studied H. pylori-positive and -negative dyspeptic patients.

\begin{tabular}{|c|c|c|c|c|c|c|}
\hline & & & No & H. pylori & $t / X^{2}$ & $\mathrm{P}$ \\
\hline & Age & & $43.31 \pm 7.41$ & $45.68 \pm 5.65$ & 2.588 & $0.01^{\star}$ \\
\hline \multirow{4}{*}{ Sex } & \multirow{2}{*}{ Male } & $\mathrm{N}$ & 152 & 68 & & \\
\hline & & $\%$ & $100.0 \%$ & $77.3 \%$ & & \\
\hline & \multirow{2}{*}{ Female } & $\mathrm{N}$ & 0 & 20 & 37.68 & $0.00^{\star *}$ \\
\hline & & $\%$ & $0.0 \%$ & $22.7 \%$ & & \\
\hline \multirow{4}{*}{ Smoking } & \multirow{2}{*}{ Non } & $\mathrm{N}$ & 112 & 68 & & \\
\hline & & $\%$ & $73.7 \%$ & $77.3 \%$ & & \\
\hline & \multirow{2}{*}{ Smoker } & $\mathrm{N}$ & 40 & 20 & 0.383 & 0.536 \\
\hline & & $\%$ & $26.3 \%$ & $22.7 \%$ & & \\
\hline \multirow{4}{*}{ Reflux } & \multirow{2}{*}{$-\mathrm{VE}$} & $\mathrm{N}$ & 80 & 56 & & \\
\hline & & $\%$ & $52.6 \%$ & $63.6 \%$ & & \\
\hline & \multirow{2}{*}{$+\mathrm{VE}$} & $\mathrm{N}$ & 72 & 32 & 2.74 & 0.097 \\
\hline & & $\%$ & $47.4 \%$ & $36.4 \%$ & & \\
\hline \multirow{4}{*}{ Hiatus hernia } & \multirow{2}{*}{$-\mathrm{VE}$} & $\mathrm{N}$ & 136 & 72 & & \\
\hline & & $\%$ & $89.5 \%$ & $81.8 \%$ & & \\
\hline & \multirow{2}{*}{$+\mathrm{VE}$} & $\mathrm{N}$ & 16 & 16 & 2.827 & 0.093 \\
\hline & & $\%$ & $10.5 \%$ & $18.2 \%$ & & \\
\hline
\end{tabular}




\section{Continued}

\begin{tabular}{|c|c|c|c|c|c|c|}
\hline \multirow{4}{*}{ Gastritis } & \multirow{2}{*}{$-\mathrm{VE}$} & $\mathrm{N}$ & 16 & 16 & \multirow{4}{*}{2.827} & \multirow{4}{*}{0.093} \\
\hline & & $\%$ & $10.5 \%$ & $18.2 \%$ & & \\
\hline & \multirow{2}{*}{$+\mathrm{VE}$} & $\mathrm{N}$ & 136 & 72 & & \\
\hline & & $\%$ & $89.5 \%$ & $81.8 \%$ & & \\
\hline \multirow{4}{*}{ Erosion } & \multirow{2}{*}{$-\mathrm{VE}$} & $\mathrm{N}$ & 128 & 56 & & \\
\hline & & $\%$ & $84.2 \%$ & $63.6 \%$ & & \\
\hline & \multirow{2}{*}{$+\mathrm{VE}$} & $\mathrm{N}$ & 24 & 32 & 13.18 & $0.00^{* *}$ \\
\hline & & $\%$ & $15.8 \%$ & $36.4 \%$ & & \\
\hline \multirow{4}{*}{ Peptic ulcer } & \multirow{2}{*}{$-\mathrm{VE}$} & $\mathrm{N}$ & 136 & 80 & & \\
\hline & & $\%$ & $89.5 \%$ & $90.9 \%$ & & \\
\hline & \multirow{2}{*}{$+\mathrm{VE}$} & $\mathrm{N}$ & 16 & 8 & 0.128 & 0.721 \\
\hline & & $\%$ & $10.5 \%$ & $9.1 \%$ & & \\
\hline \multirow{4}{*}{$\begin{array}{c}\text { Mucosal } \\
\text { nodularity }\end{array}$} & \multirow{2}{*}{$-\mathrm{VE}$} & $\mathrm{N}$ & 144 & 80 & & \\
\hline & & $\%$ & $94.7 \%$ & $90.9 \%$ & & \\
\hline & \multirow{2}{*}{$+\mathrm{VE}$} & $\mathrm{N}$ & 8 & 8 & 1.31 & 0.252 \\
\hline & & $\%$ & $5.3 \%$ & $9.1 \%$ & & \\
\hline \multirow{4}{*}{$\begin{array}{l}\text { Chronic active } \\
\text { gastritis }\end{array}$} & \multirow{2}{*}{$-\mathrm{VE}$} & $\mathrm{N}$ & 84 & 48 & & \\
\hline & & $\%$ & $55.3 \%$ & $54.5 \%$ & & \\
\hline & \multirow{2}{*}{$+\mathrm{VE}$} & $\mathrm{N}$ & 68 & 40 & 0.012 & 0.914 \\
\hline & & $\%$ & $44.7 \%$ & $45.5 \%$ & & \\
\hline \multirow{4}{*}{$\begin{array}{l}\text { Chronic } \\
\text { inactive } \\
\text { gastritis }\end{array}$} & \multirow{2}{*}{$-\mathrm{VE}$} & $\mathrm{N}$ & 68 & 48 & & \\
\hline & & $\%$ & $44.7 \%$ & $54.5 \%$ & & \\
\hline & \multirow{2}{*}{$+\mathrm{VE}$} & $\mathrm{N}$ & 84 & 40 & 2.147 & 0.143 \\
\hline & & $\%$ & $55.3 \%$ & $45.5 \%$ & & \\
\hline \multirow{4}{*}{$\begin{array}{l}\text { Intestinal } \\
\text { metaplasia }\end{array}$} & \multirow{2}{*}{$-\mathrm{VE}$} & $\mathrm{N}$ & 148 & 84 & & \\
\hline & & $\%$ & $97.4 \%$ & $95.5 \%$ & & \\
\hline & \multirow{2}{*}{$+\mathrm{VE}$} & $\mathrm{N}$ & 4 & 4 & 0.63 & 0.42 \\
\hline & & $\%$ & $2.6 \%$ & $4.5 \%$ & & \\
\hline \multirow{4}{*}{$\begin{array}{l}\text { Erosive } \\
\text { gastritis }\end{array}$} & \multirow{2}{*}{$-\mathrm{VE}$} & $\mathrm{N}$ & 124 & 60 & & \\
\hline & & $\%$ & $81.6 \%$ & $68.2 \%$ & & \\
\hline & VI & $\mathrm{N}$ & 28 & 28 & 5.59 & $0.018^{*}$ \\
\hline & 1.2 & $\%$ & $18.4 \%$ & $31.8 \%$ & & \\
\hline & VE & $\mathrm{N}$ & 140 & 84 & & \\
\hline Glandular & & $\%$ & $92.1 \%$ & $95.5 \%$ & & \\
\hline atrophy & & $\mathrm{N}$ & 12 & 4 & 1.00 & 0.31 \\
\hline & TV & $\%$ & $7.9 \%$ & $4.5 \%$ & & \\
\hline & $-\mathrm{VE}$ & $\mathrm{N}$ & 148 & 84 & & \\
\hline Reactive & & $\%$ & $97.4 \%$ & $95.5 \%$ & & \\
\hline gastropathy & UT & $\mathrm{N}$ & 4 & 4 & 0.634 & 0.426 \\
\hline & 10 & $\%$ & $2.6 \%$ & $4.5 \%$ & & \\
\hline & & $\mathrm{N}$ & 152 & 88 & & \\
\hline & & $\%$ & $100.0 \%$ & $100.0 \%$ & & \\
\hline
\end{tabular}


Table 6. Relation between the studied dyspeptic $H$. pylori-positive smokers and endoscopic and histological finding.

\begin{tabular}{|c|c|c|c|c|c|c|c|}
\hline & & & H. pylori & positive & & & \\
\hline & & & Non smoker & Smoker & & & \\
\hline & & $\mathrm{N}$ & 12 & 8 & 20 & & \\
\hline & $-V E$ & $\%$ & $30.0 \%$ & $40.0 \%$ & $33.3 \%$ & & \\
\hline Nerus & & $\mathrm{N}$ & 28 & 12 & 40 & 0.60 & 0.439 \\
\hline & $+V E$ & $\%$ & $70.0 \%$ & $60.0 \%$ & $66.7 \%$ & & \\
\hline & $-\mathrm{VF}$ & $\mathrm{N}$ & 32 & 20 & 52 & & \\
\hline & $-V L$ & $\%$ & $80.0 \%$ & $100.0 \%$ & $86.7 \%$ & & \\
\hline Hifatus nerinda & & $\mathrm{N}$ & 8 & 0 & 8 & 4.61 & $0.032^{\star}$ \\
\hline & $+V E$ & $\%$ & $20.0 \%$ & $0.0 \%$ & $13.3 \%$ & & \\
\hline & $-\mathrm{VE}$ & $\mathrm{N}$ & 8 & 8 & 16 & & \\
\hline & $-v \mathrm{~L}$ & $\%$ & $20.0 \%$ & $40.0 \%$ & $26.7 \%$ & & \\
\hline Gastitis & & $\mathrm{N}$ & 32 & 12 & 44 & 2.72 & 0.099 \\
\hline & $+\mathrm{VE}$ & $\%$ & $80.0 \%$ & $60.0 \%$ & $73.3 \%$ & & \\
\hline & $-\mathrm{VE}$ & $\mathrm{N}$ & 28 & 8 & 36 & & \\
\hline & & $\%$ & $70.0 \%$ & $40.0 \%$ & $60.0 \%$ & & \\
\hline & & $\mathrm{N}$ & 12 & 12 & 24 & 5.0 & $0.025^{\star}$ \\
\hline & TV & $\%$ & $30.0 \%$ & $60.0 \%$ & $40.0 \%$ & & \\
\hline & $\mathrm{VF}$ & $\mathrm{N}$ & 36 & 12 & 48 & & \\
\hline & $v_{L}$ & $\%$ & $90.0 \%$ & $60.0 \%$ & $80.0 \%$ & & \\
\hline & & $\mathrm{N}$ & 4 & 8 & 12 & 7.50 & $0.006^{\star}$ \\
\hline & $T V L$ & $\%$ & $10.0 \%$ & $40.0 \%$ & $20.0 \%$ & & \\
\hline & $-\mathrm{VE}$ & $\mathrm{N}$ & 32 & 16 & 48 & & \\
\hline Mucosal & & $\%$ & $80.0 \%$ & $80.0 \%$ & $80.0 \%$ & & \\
\hline nodularity & & $\mathrm{N}$ & 8 & 4 & 12 & 0.00 & 1.00 \\
\hline & & $\%$ & $20.0 \%$ & $20.0 \%$ & $20.0 \%$ & & \\
\hline & & $\mathrm{N}$ & 20 & 8 & 28 & & \\
\hline $\begin{array}{l}\text { Chronic } \\
\text { active }\end{array}$ & $-V E$ & $\%$ & $50.0 \%$ & $40.0 \%$ & $46.7 \%$ & & \\
\hline gastritis & $+\mathrm{VE}$ & $\mathrm{N}$ & 20 & 12 & 32 & 0.536 & 0.464 \\
\hline & & $\%$ & $50.0 \%$ & $60.0 \%$ & $53.3 \%$ & & \\
\hline & & $\mathrm{N}$ & 20 & 20 & 40 & & \\
\hline Chronic & $-\mathrm{VE}$ & $\%$ & $50.0 \%$ & $100.0 \%$ & $66.7 \%$ & & \\
\hline gastritis & $+\mathrm{VF}$ & $\mathrm{N}$ & 20 & 0 & 20 & 15.0 & $0.00^{* *}$ \\
\hline & & $\%$ & $50.0 \%$ & $0.0 \%$ & $33.3 \%$ & & \\
\hline & & $\mathrm{N}$ & 40 & 16 & 56 & & \\
\hline Intestinal & & $\%$ & $100.0 \%$ & $80.0 \%$ & $93.3 \%$ & & \\
\hline metaplasia & & $\mathrm{N}$ & 0 & 4 & 4 & 8.57 & $0.003^{*}$ \\
\hline & & $\%$ & $0.0 \%$ & $20.0 \%$ & $6.7 \%$ & & \\
\hline
\end{tabular}




\begin{tabular}{|c|c|c|c|c|c|c|c|}
\hline \multicolumn{8}{|l|}{ Continued } \\
\hline \multirow{4}{*}{$\begin{array}{l}\text { Erosive } \\
\text { gastritis }\end{array}$} & \multirow{4}{*}{$\begin{array}{l}-\mathrm{VE} \\
+\mathrm{VE}\end{array}$} & $\mathrm{N}$ & 28 & 4 & 32 & \multirow{4}{*}{13.39} & \multirow{4}{*}{$0.00^{\star *}$} \\
\hline & & $\%$ & $70.0 \%$ & $20.0 \%$ & $53.3 \%$ & & \\
\hline & & $\mathrm{N}$ & 12 & 16 & 28 & & \\
\hline & & $\%$ & $30.0 \%$ & $80.0 \%$ & $46.7 \%$ & & \\
\hline \multirow{4}{*}{$\begin{array}{c}\text { Grandular } \\
\text { atrophy }\end{array}$} & \multirow{2}{*}{$-\mathrm{VE}$} & $\mathrm{N}$ & 40 & 16 & 56 & \multirow{4}{*}{8.57} & \multirow{4}{*}{$0.003^{\star}$} \\
\hline & & $\%$ & $100.0 \%$ & $80.0 \%$ & $93.3 \%$ & & \\
\hline & \multirow{2}{*}{$+\mathrm{VE}$} & $\mathrm{N}$ & 0 & 4 & 4 & & \\
\hline & & $\%$ & $0.0 \%$ & $20.0 \%$ & $6.7 \%$ & & \\
\hline \multirow{4}{*}{$\begin{array}{c}\text { Reactive } \\
\text { gastropathy }\end{array}$} & \multirow{2}{*}{$-\mathrm{VE}$} & $\mathrm{N}$ & 40 & 16 & 56 & \multirow{4}{*}{8.57} & \multirow{4}{*}{$0.003^{\star}$} \\
\hline & & $\%$ & $100.0 \%$ & $80.0 \%$ & $93.3 \%$ & & \\
\hline & \multirow{2}{*}{$+\mathrm{VE}$} & $\mathrm{N}$ & 0 & 4 & 4 & & \\
\hline & & $\%$ & $0.0 \%$ & $20.0 \%$ & $6.7 \%$ & & \\
\hline \multirow{2}{*}{\multicolumn{2}{|c|}{ Total }} & $\mathrm{N}$ & 40 & 20 & 60 & & \\
\hline & & $\%$ & $100.0 \%$ & $100.0 \%$ & $100.0 \%$ & & \\
\hline
\end{tabular}

\section{Discussion}

In the current study, the prevalence of $H$. pylori positivity among dyspeptic patients was $36.7 \%$. These results are consistent with those of another Egyptian study by Khalifa et al., in which the prevalence of $H$. pylori positivity was $43 \%$ [22]. However, a study in North Sulawesi and Indonesia, reported lower H. pylori prevalence in patients referred for endoscopy (14.3\%) [23]. These differences in prevalence may be due to the overcrowded conditions and poor socioeconomic status [24] as in Egypt, it has been reported that the prevalence of $H$. $p y$ lori infection is $90 \%$ in adults [25]. We found that $H$. pylori infection was most commonly observed in adults with an average age of 46 years which is in accordance with the result of Basilio et al. [26]. This may be explained by an increase the prevalence of infection in the community with increase the age [27]. Furthermore, aging is linked to a reduced turnover rate of epithelial cells as well as the potential for gastric mucosa repair [28].

Endoscopy revealed mostly gastritis, esophageal reflux or both in our study. This is similar to what has been described by Vasiliou et al. who showed that the major endoscopic findings among 160 patients with dyspeptic were chronic gastritis (83.8\%), and erosive esophagitis (34.4\%) [29]. moreover, the prevalence of gastritis, in a similar study, of Sahin et al. was $48.4 \%$ [30]. However, in another Egyptian study, irrelevant findings in $65 \%$, peptic ulcer in 18\%, esophagitis in $14 \%$ and erosive gastroduodenitis in $8 \%$ were detected during endoscopy of patients presenting with dyspepsia where $82 \%$ of these patients younger than 30 years [31]. This controversy among both of Egyptian studies may be due to the difference in age between the studied patients.

Surprisingly, the researchers have detected contradictory results about the relation between $H$. pylori and smoking. We found no statistically significant difference between smokers, non-smokers in the positivity rate of $H$. pylori $(\mathrm{P}=$ 
0.536) and similar results have been reported by Khalifa et al. among dyspepsia Egyptian patients [22]. In contrary to the findings of Rajashekhar et al. who had found an association of smoking with $H$. pylori positivity in dyspepsia patients [32]. The latter finding is not in line with the current data which demonstrate that $H$. pylori infection acquired during childhood when smoking has no role [33].

In this study, the number of the smoker was higher among men, which is expected in our community [34]. In addition, there was statistically significant difference in the endoscopic findings including; reflux, erosion, peptic ulcer and mucosal nodularity between smokers and non-smokers. Our findings are in agreement with those of Namiot et al. who observed that most ulcer disease patients were men and smokers [35]. Regarding histological examination, smoker patients were statistically significantly higher only in erosive gastritis. Our results concur well with another study by El Hamshary et al. conducted in Fayoum governorate that demonstrated a highly significant association between chronic gastritis and smoking habit [36]. This may be due to aggressive gastric factors (such as duodenogastric reflux acid, pepsin secretion, and free-radical production) and impair mucosal defenses (such as prostaglandin synthesis, mucus production, and epidermal growth factor secretion) which are potentiated by nicotine [37].

Moreover, we found that $H$. pylori-positive patients showed more erosion and erosive gastritis compared to $H$. pylori-negative patients and we support the results of Banik et al. who detected that erosive gastritis and non-erosive gastritis patients were $H$. pylori sero-positive [38]. We observed that dyspeptic $H$. pylo$r i$-positive smokers more likely had erosion and peptic ulcer than dyspeptic $H$. pylori-positive non-smokers. This result is consistent with the finding of Martin et al. who reported that smokers with positive $H$. pylori were more likely to have a peptic or duodenal ulcer (73\%) compared to $H$. pylori-positive nonsmokers (29\%) [39]. Also, McColl et al. have demonstrated that smokers with positive $H$. pylori were more likely to have a peptic or duodenal ulcer (67\%) than $\mathrm{H}$. pylori-positive nonsmokers (46\%) [40]. Koivisto et al., on the other hand found that smoking reduced gastric body inflammation and atrophy as well as humeral response to $H$. pylori among $H$. pylori-positive patients with gastritis [41]. As regard histological findings, dyspeptic $H$. pylori-positive smokers developed more intestinal metaplasia, erosive gastritis, glandular atrophy and reactive gastropathy rather than dyspeptic $H$. pylori-positive non-smokers. These results were in agreement with Russo et al. who found that among $H$. pylori-positive patients, there was a strong association amongst gastric IM and smoking [42]. Nakamura et al. also found that In $H$. pylori positive patients, smoking can play a key role in the pathogenesis of IM and AG [43]. An in vitro research has also revealed that nicotine enhanced the activity of $H$. pylori vacuolating toxin [44].

\section{Limitations of the Study}

Firstly, this research was conducted on a small number of population samples 
with no matching groups according to sex and smoking habit as all of the smokers were men and none of females were smokers according to their cultural aspect. Secondly, we didn't classify the smokers according to its smoking intensity and duration to be compared with the endoscopic and histological finding of them.

\section{Conclusion}

On the basis of the findings of the present study, there is no association between $H$. pylori infection and smoking. However, we confirmed that smoking triggered endoscopic finding including gastritis and histological changes were associated with $H$. pylori infection including intestinal metaplasia, erosive gastritis, glandular atrophy and reactive gastropathy.

\section{Acknowledgements}

Thanks to members of endoscopic units in the Tropical Medicine Department of both Zagazig and Tanta University Hospital, Egypt who participated and cooperated in the study.

\section{Conflicts of Interest}

The authors declare no conflicts of interest regarding the publication of this paper.

\section{References}

[1] Mapel, D., Roberts, M., Overhiser, A. and Mason, A. (2013) The Epidemiology, Diagnosis, and Cost of Dyspepsia and Helicobacter pylori Gastritis: A Case-Control Analysis in the Southwestern United States. Helicobacter, 18, 54-65. https://doi.org/10.1111/j.1523-5378.2012.00988.x

[2] Tack, J., Talley, N.J., Camilleri, M., Holtmann, G., Hu, P., Malagelada, J.R. and Stanghellini, V. (2006) Functional Gastroduodenal Disorders. Gastroenterology, 130, 1466-1479. https://doi.org/10.1053/j.gastro.2005.11.059

[3] Al-Humayed, S.M., Mohammed-Elbagir, A.K., Al-Wabel, A.A. and Argobi, Y.A. (2010) The Changing Pattern of Upper Gastrointestinal Lesions in Southern Saudi Arabia: An Endoscopic Study. Saudi Journal of Gastroenterology, 16, 35-37. https://doi.org/10.4103/1319-3767.58766

[4] Aziz, A.F.A., Hamzah, Z., Tong, S.F., Nadeson, S. and Wan Puteh, S.E. (2009) Helicobacter pylori Related Dyspepsia: Prevalence and Treatment Outcomes at University Kebangsaan Malaysia-Primary Care Centre. Asia Pacific Family Medicine, 8, Article No. 4. https://doi.org/10.1186/1447-056X-8-4

[5] Olokoba, A.B., Obateru, O.A., Bojuwoye, M.O., Ibrahim, O.K. and Olokoba, L.B. (2013) That Dyspepsia in the Young Could Be Cancer. Nigerian Medical Journal, 54, 143-145. https://doi.org/10.4103/0300-1652.110053

[6] Mahadeva, S. and Goh, K.-L. (2006) Epidemiology of Functional Dyspepsia: A Global Perspective. World Journal of Gastroenterology, 12, 2661-2666. https://doi.org/10.3748/wjg.v12.i17.2661

[7] Ford, A.C., Marwaha, A., Sood, R. and Moayyedi, P. (2015) Global Prevalence of, and Risk Factors for, Uninvestigated Dyspepsia: A Meta-Analysis. Gut, 64, 1049-1057. https://doi.org/10.1136/gutjnl-2014-307843 
[8] Flier, S.N. and Rose, S. (2006) Is Functional Dyspepsia of Particular Concern in Women? A Review of Gender Differences in Epidemiology, Pathophysiologic Mechanisms, Clinical Presentation, and Management. The American Journal of Gastroenterology, 101, S644-S653.

[9] Ardakani, A. and Mohammadizadeh, F. (2006) The Study of Relationship between Helicobacter pylori Density in Gastric Mucosaand the Severity and Activity of Chronic Gastritis. Journal of Research in Medical Sciences, 11, 282.

[10] Go, M.F. (2002) Review Article: Natural History and Epidemiology of Helicobacter pylori Infection. Alimentary Pharmacology \& Therapeutics, 16, 3-15. https://doi.org/10.1046/j.1365-2036.2002.0160s1003.x

[11] Rowland, M., Daly, L., Vaughan, M., Higgins, A., Bourke, B. and Drumm, B. (2006) Age-Specific Incidence of Helicobacter pylori. Gastroenterology, 130, 65-72.

https://doi.org/10.1053/j.gastro.2005.11.004

[12] Hocker, M. and Hohenberger, P. (2003) Helicobacter pylori Virulence Factors-One Part of a Big Picture. The Lancet, 362, 1231-1233.

https://doi.org/10.1016/S0140-6736(03)14547-3

[13] Dixon, M.F., Genta, R.M., Yardley, J.H. and Correa, P. (1996) Classification and Grading of Gastritis. The Updated Sydney System. International Workshop on the Histopathology of Gastritis, Houston 1994. The American Journal of Surgical Pathology, 20, 1161-1181. https://doi.org/10.1097/00000478-199610000-00001

[14] Yang, S., Valvo, S., Yao, H., Kode, A., Rajendrasozhan, S., Edirisinghe, I., et al. (2008) IKKa Causes Chromatin Modification on Pro-Inflammatory Genes by Cigarette Smoke in Mouse Lung. American Journal of Respiratory Cell and Molecular Biology, 38, 689-698. https://doi.org/10.1165/rcmb.2007-0379OC

[15] Zhang, L., Ren, J.W., Wong, C.C., Wu, W.K., Ren, S.X., Shen, J., et al. (2012) Effects of Cigarette Smoke and Its Active Components on Ulcer Formation and Healing in the Gastrointestinal Mucosa. Current Medicinal Chemistry, 19, 63-69. https://doi.org/10.2174/092986712803413926

[16] Ogihara, A., Kikuchi, S., Hasegawa, A., Kurosawa, M., Miki, K., Kaneko, E. and Mizukoshi, H. (2000) Relationship between Helicobacter pylori Infection and Smoking and Drinking Habits. Journal of Gastroenterology and Hepatology, 15, 271-276. https://doi.org/10.1046/j.1440-1746.2000.02077.x

[17] Li, L.F., Chan, R.L., Lu, L., Shen, J., Zhang, L., Wu, W.K., et al. (2014) Cigarette Smoking and Gastrointestinal Diseases: The Causal Relationship and Underlying Molecular Mechanisms (Review). International Journal of Molecular Medicine, 34, 372-380. https://doi.org/10.3892/ijmm.2014.1786

[18] Stemmermann, G.N., Nomura, A.M.Y., Chyou, P.-H. and Hankin, J. (1990) Impact of Diet and Smoking on Risk of Developing Intestinal Metaplasia of the Stomach. Digestive Diseases and Sciences, 35, 433-438. https://doi.org/10.1007/BF01536915

[19] Ozasa, K., Kurata, J.H., Higashi, A., Hayashi, K., Inokuchi, H., Miki, K., et al. (1999) Helicobacter pylori Infection and Atrophic Gastritis: A Nested Case-Control Study in Rural Town in Japan. Digestive Diseases and Sciences, 44, 253-256.

[20] Shousha, S., Barrison, I.G., EI Sayeed, W., Kahn, S. and Parkins, R.A. (1984) A Study of Incidence and Relationship of Intestinal Metaplasia of Gastric Antrum and Gastric Metaplasia of Gastric Antrum and Gastric Metaplasia of the Duodenum in Patients with Non-Ulcer Dyspepsia. Digestive Diseases and Sciences, 29, 311-316. https://doi.org/10.1007/BF01318515

[21] Kneller, R.W., You, W.C., Chang, Y.S., Liu, W.D., Zhang, L., Zhao, L., et al. (1992) Cigarette Smoking and Other Risk Factors for Progression of Precancerous Sto- 
mach Lesions. Journal of the National Cancer Institute, 84, 1261-1266.

https://doi.org/10.1093/jnci/84.16.1261

[22] Khalifa, M., Khodiar, S. and Abd Almaksoud, A. (2014) Cigarette Smoking Status and Helicobacter pylori Infection in Non-Ulcer Dyspepsia Patients. Egyptian Journal of Chest Diseases and Tuberculosis, 63, 695-699.

https://doi.org/10.1016/j.ejcdt.2014.03.007

[23] Mohammad, M., Josef, T., Rumiko, S., Yasutoshi, K., Fumihiko, K., Miyuki, M., et al. (2014) Extremely Low Helicobacter pylori Prevalence in North Sulawesi, Indonesia and Identification of a Maori-Tribe Type Strain: A Cross Sectional Study. Gut Pathogens, 6, Article No. 42. https://doi.org/10.1186/s13099-014-0042-0

[24] Cheng, H., Hu, F., Zhang, L., Yang, G., Ma, J., Hu, J., et al. (2009) Prevalence of Helicobacter pylori Infection and Identification of Risk Factors in Rural and Urban Beijing, China. Helicobacter, 14, 128-133.

https://doi.org/10.1111/j.1523-5378.2009.00668.x

[25] Hunt, R.H., Xiao, S.D., Megraud, F., Leon-Barua, R., Bazzoli, F. and van der Merwe, S. (2011) Helicobacter pylori in Developing Countries World Gastroenterology Organization Global Guideline. Journal of Gastrointestinal and Liver Diseases, 20, 299-304.

[26] Basílio, I.L.D., Catão, M.F.C., Carvalho, J.D.S., Freire-Neto, F.P., Ferreira, L.C. and Jerônimo, S.M.B. (2018) Risk Factors of Helicobacter pylori Infection in an Urban Community in Northeast Brazil and the Relationship between the Infection and Gastric Diseases. Revista da Sociedade Brasileira de Medicina Tropical, 51, 183-189. https://doi.org/10.1590/0037-8682-0412-2016

[27] Poddar, U. and Yachha, S.K. (2007) Helicobacter pylori in Children: An Indian Perspective. Indian Pediatrics, 44, 761-770.

[28] Pilotto, A. and Franceschi, M. (2014) Helicobacter pylori Infection in Older People. World Journal of Gastroenterology, 20, 6364-6373. https://doi.org/10.3748/wjg.v20.i21.6364

[29] Vasiliou, C., Xiromeritou, V., Kafiri, G. and Papatheodoridis, G. (2014) Endoscopic and Histological Findings and Helicobacter pylori Status in Patients with Reflux and/or Dyspeptic Symptoms: A Recent Greek Cohort Study. Gastroenterology Nursing, 37, 431-438. https://doi.org/10.1097/SGA.0000000000000075

[30] Sahin, M., Akbulut, C., Dolapcioglu, C., Ozpolat, E., Dabak, R., Aliustaoğlu, M., et al. (2014) Endoscopic Findings of Dyspeptic Patients Unresponsive to Proton Pump Inhibitors. Northern Clinics of Istanbul, 1, 158-165.

https://doi.org/10.14744/nci.2014.54227

[31] Gadoa, A., Ebeidb, B., Abdelmohsen, A. and Axond, A. (2015) Endoscopic Evaluation of Patients with Dyspepsia in a Secondary Referral Hospital in Egypt. Alexandria Journal of Medicine, 51, 179-184. https://doi.org/10.1016/j.ajme.2013.10.001

[32] Rajashekhar, V., Bhasin, D.K., Ray, P., Vaiphei, K., Sharma, B.C. and Singh, K. (2000) Helicobacter pylori Infection in Chronic Smokers with Non-Ulcer Dyspepsia. Tropical Gastroenterology, 21, 71-72.

[33] Koletzko, S., Jones, N.L., Goodman, K.J., Gold, B., Rowland, J., Cadranel, S., et al. (2011) H. pylori Working Groups of ESPGHAN and NASPGHAN. Evidence-Based Guidelines from ESPGHAN and NASPGHAN for Helicobacter pylori Infection in Children. Journal of Pediatric Gastroenterology and Nutrition, 53, 230-243. https://doi.org/10.1097/MPG.0b013e3182227e90

[34] World Health Organization (2015) WHO Global Report on Trends in Prevalence of Tobacco Smoking 2015. World Health Organization, Geneva. 
[35] Namiot, A., Kemona, A. and Namiot, Z. (2007) Smoking Habit and Gastritis Histology. Advances in Medical Sciences, 52, 191-195.

[36] El Hamshary, N.K., ALmetwaly, M.T., El-Shafie, A.M., Kelani, Y. and Eltiby, D. (2011) Study of the Risk Factors of Chronic Gastritis in Fayoum Governorate. $A A M J, 9,1$.

[37] Endoh, K. and Leung, F.W. (1994) Effects of Smoking and Nicotine on Gastric Mucosa: A Review of Clinical and Experimental Evidence. Gastroenterology, 107, 864-878. https://doi.org/10.1016/0016-5085(94)90138-4

[38] Banik, T., Bar, P.K. and Mandal, S. (2020) Helicobacter pylori Chronic Gastritis: Correlation between Endoscopic Findings and Histopathology with Special Reference to Updated Sydney System. International Journal of Medical Research and Review, 8, 7-13. https://doi.org/10.17511/ijmrr.2020.i01.02

[39] Martin, D.F., Montgomery, E., Dobek, A.S., Patrissi, G.A. and Peura, D.A. (1989) Campylobacter pylori, NSAIDS, and Smoking: Risk Factors for Peptic Ulcer Disease. American Journal of Gastroenterology, 84, 1268-1272.

[40] Labenz, J., Stolte, M., Blum, A.L., Jorias, I., Leverkus, F., Sollbohmer, M., et al. (1995) Intragastric Acidity as a Predictor of the Success of Helicobacter pylori Eradication: A Study in Peptic Ulcer Patients with Omeprazole and Amoxicillin. Gut, 37, 39-43. https://doi.org/10.1136/gut.37.1.39

[41] Koivisto, T.T., Voutilainen, M.E. and Färkkilä, M.A. (2008) Symptoms, Endoscopic Findings and Histology Predicting Symptomatic Benefit of Helicobacter pylori Eradication. Scandinavian Journal of Gastroenterology, 43, 810-816. https://doi.org/10.1080/00365520801935426

[42] Russo, A., Maconi, G., Spinelli, P., Felice, G.D., Eboli, M., Andreola, S., et al. (2001) Effect of Lifestyle, Smoking, and Diet on Development of Intestinal Metaplasia in $H$. pylori-positive Subjects. American Journal of Gastroenterology, 96, 1402-1408.

[43] Nakamura, M., Haruma, K., Kamada, T., Mihara, M., Yoshihara, M., Sumioka, M., et al. (2002) Cigarette Smoking Promotes Atrophic Gastritis in Helicobacter pylori-positive Subjects. Digestive Diseases and Sciences, 47, 675-681. https://doi.org/10.1023/A:1017901110580

[44] Cover, T.L., Vaughn, S.G., Cao, P. and Blaser, M.J. (1992) Potentiation of Helicobacter pylori Vacuolating Toxin Activity by Nicotine and Other Weak Bases. The Journal of Infectious Diseases, 166, 1073-1078. https://doi.org/10.1093/infdis/166.5.1073 